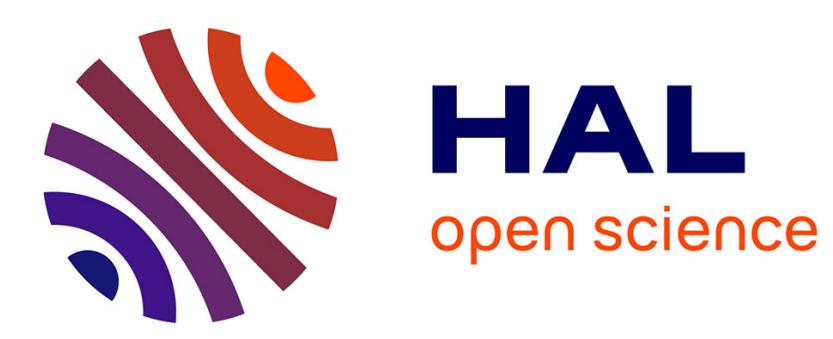

\title{
Estimation of the Hurst parameter in some fractional processes
}

Luis Armando Salomon, Jean-Claude Fort

\section{To cite this version:}

Luis Armando Salomon, Jean-Claude Fort. Estimation of the Hurst parameter in some fractional processes. 2011. hal-00626693

\section{HAL Id: hal-00626693 \\ https://hal.science/hal-00626693}

Preprint submitted on 26 Sep 2011

HAL is a multi-disciplinary open access archive for the deposit and dissemination of scientific research documents, whether they are published or not. The documents may come from teaching and research institutions in France or abroad, or from public or private research centers.
L'archive ouverte pluridisciplinaire HAL, est destinée au dépôt et à la diffusion de documents scientifiques de niveau recherche, publiés ou non, émanant des établissements d'enseignement et de recherche français ou étrangers, des laboratoires publics ou privés. 


\title{
Estimation of the Hurst parameter in some fractional processes
}

\author{
Luis Salomon ${ }^{\mathrm{a}, \mathrm{b}}$, Jean-Claude Fort ${ }^{\mathrm{c}}$ \\ a "Université de la Havane" \\ ${ }^{b}$ Institut de Mathématiques de Toulouse, 118 route de Narbonne F-31062 Toulouse \\ ${ }^{c}$ Université Paris Descartes, 45 rue des saints pères, 75006 Paris, France
}

\begin{abstract}
We propose to estimate the Hurst parameter involved in fractional processes via a method based on the Karhunen-Loève expansion of Gaussian process. We specifically investigate the cases of the Fractional Brownian motion(FBm) and the Fractional Ornstein-Uhlenbeck(FOU) Family. The main tool is the analysis of the residuals of a convenient linear regression model. We numerically compare our results with the ones obtained by the maximum likelihood method, which show the validity of our proposal.
\end{abstract}

Key words: ,,

\section{Introduction}

Our goal is to propose an estimator of the hurst parameter of a fractional gaussian process that performs almost as the maximum likelihood estimator but is more robust to computational problems.

Our framework is quite simple : we have observations of a random process at equally spaced or randomly distributed times, $X\left(t_{1}\right), \ldots, X\left(t_{n}\right)$. Assuming that the model which rules these observations is a gaussian fractional process (Brownian motion, Ornstein Ulhenbeck process, Brownian bridge) we estimate the hurst parameter denoted by $\theta, \theta \in(0,1)$ ]. We are strictly in the parametric estimation framework.

Obviously the best estimator is obtain by maximizing the likelihood. It involves the numerical computation of the inverse of the covariance matrix, which is tedious when $n$ is of magnitude larger than 500, even using $L U$ decomposition.

Our alternative method relies on the Karhune-Loève decomposition, but in practice only needs a short expansion, say less than 10 terms.

The next section presents our framework and recall some basic results about KarhuneLoève decomposition. In section 3 we define our estimator and give some asymptotic results that prove its consistency. Finally a section is devoted to numerical comparison with the Maximum Likelihood Estimator (M.L.E.) on several examples. Fort)

URL: salo@matcom.uh.cu (Luis Salomon), jean-claude.fort@parisdescartes.fr (Jean-Claude 


\section{Framework and basic results}

In this section we introduce a method based in the Karhunen-Loève expansion of Gaussian process to estimate the Hurst parameter involved in fractional processes, specifically the Fractional Brownian motion(FBm) and the Fractional Ornstein-Uhlenbeck(FOU) Family. Our aim is to compare our results with the ones obtained by the maximum likelihood method and show the validity of our proposal.

In the sequel for all the fractional process we will consider, we deal with only one unknown parameter of interest: the hurst parameter $\theta$. This parameter describes the roughness of the path we partially observe.

In many cases other parameters are to be considered as mean, variance, drift parameter... We will discuss these cases further on.

For instance for the Fractional Ornstein-Ulhenbeck family we assume that $\mu=0$ and that $\alpha$ the drift parameter and $\sigma$ the spatial scale are known.

\subsubsection{Karhune-Loève decomposition}

We will consider from now on that $X$ belongs to one of the family $\left(X^{\theta}\right)$ of centered fractional Gaussian process mentioned before with Hurst parameter $\theta$ in $[0,1]$. We assume that the observation time interval is $[0,1]$. Let us denote by $\theta_{0}$ the real and unknown parameter of the process actually observe, $X_{0}^{\theta}$. It is well-known that a Gaussian process has a unique representation in the basis of Karhunen-Loève. This basis is given by the diagonalisation of the so-called covariance operator $\Gamma$ of the process $\mathrm{X}$, which is assumed to be a Hilbert-schmidt operator:

$$
\Gamma^{\theta}(f)(t)=\mathbb{E}\left(f\left(X^{\theta}(s)\right) X^{\theta}(t) X^{\theta}(s)\right)=\int_{\mathbb{R}} f(t) \operatorname{cov}\left(X^{\theta}(t), X^{\theta}(s)\right) d s .
$$

For each value of $\theta$ we denote by $\left(\lambda_{i}^{\theta}, \varphi_{i}^{\theta}\right)_{i \geq 1}$ the eigenvalues and associated normalized eigenfunctions of $\Gamma^{\theta}$ in decreasing order. Then there exists a an i.i.d. sequence of standard normal random variables $\xi_{i}^{\theta}$, such that in $\mathcal{L}^{2}[0,1]$ :

$$
X^{\theta}(t)=\sum_{i \geq 1} \sqrt{\lambda_{i}^{\theta}} \xi_{i}^{\theta} \varphi_{i}^{\theta}(t)
$$

This is the Karhunen-Loève decomposition of $X^{\theta}$.

For $N$ an integer, splitting the sum in equation (1) we have:

$$
\begin{aligned}
X^{\theta}(t) & =\sum_{i \geq 1} \sqrt{\lambda_{i}^{\theta}} \xi_{i}^{\theta} \varphi_{i}^{\theta}(t) \\
& =\sum_{j=1}^{N} \sqrt{\lambda_{i}^{\theta}} \xi_{i}^{\theta} \varphi_{i}^{\theta}(t)+\sum_{j \geq N+1} \sqrt{\lambda_{i}^{\theta}} \xi_{i}^{\theta} \varphi_{i}^{\theta}(t) .
\end{aligned}
$$

This can be seen as a linear regression model with noise

$$
\varepsilon^{\theta}(t)=\sum_{j \geq N+1}\left\langle X^{\theta}, \varphi_{j}^{\theta}\right\rangle \varphi_{j}^{\theta}(t)=\sum_{j \geq N+1} \sqrt{\lambda_{j}^{\theta}} \xi_{j}^{\theta} \varphi_{j}^{\theta}(t),
$$


so that we may write :

$$
\begin{aligned}
X^{\theta}(t) & =\sum_{j=1}^{N} \sqrt{\lambda_{i}^{\theta}} \xi_{i}^{\theta} \varphi_{i}^{\theta}(t)+\varepsilon^{\theta}(t) \\
& =\sum_{1 \leq j \leq N}\left\langle X^{\theta}, \varphi_{j}^{\theta}\right\rangle \varphi_{j}^{\theta}(t)+\varepsilon^{\theta}(t) .
\end{aligned}
$$

The covariance function of $\varepsilon^{\theta}(t)$ is given by

$$
C^{\theta}(t, s)=\mathbb{E}_{\theta}\left(\varepsilon^{\theta}(t) \varepsilon^{\theta}(s)\right)=\sum_{j \geq N+1} \lambda_{j}^{\theta} \varphi_{j}^{\theta}(t) \varphi_{j}^{\theta}(s) .
$$

\subsection{The observations}

We assume observing the path of the process $X^{\theta_{0}}$ at $n$ times $t_{1}, \ldots, t_{n}$, which could be regularly spaced or uniformly distributed. Let $\mathbf{t}=\left(t_{1}, \cdots, t_{n}\right)^{\prime}$, in view of (3) we write:

$$
X^{\theta}(\mathbf{t})=\left(\begin{array}{c}
X^{\theta}\left(t_{1}\right) \\
\vdots \\
X^{\theta}\left(t_{n}\right)
\end{array}\right)=M_{N}^{\theta}(\mathbf{t}) \Xi_{N}^{\theta}+\varepsilon_{N}^{\theta}(\mathbf{t})
$$

where

$$
M_{N}^{\theta}(\mathbf{t})=\left(\left\langle X^{\theta}, \varphi_{j}^{\theta}\right\rangle\right)_{\substack{1 \leq i \leq n \\
1 \leq j \leq N}}, \quad \Xi_{N}^{\theta}=\left(\begin{array}{c}
\xi_{1}^{\theta} \\
\vdots \\
\xi_{N}^{\theta}
\end{array}\right), \quad \varepsilon_{N}^{\theta}(\mathbf{t})=\left(\begin{array}{c}
\varepsilon^{\theta}\left(t_{1}\right) \\
\vdots \\
\varepsilon^{\theta}\left(t_{n}\right)
\end{array}\right),
$$

This is a classical multiple regression model:

$$
X^{\theta}(\mathbf{t})=M_{N}^{\theta}(\mathbf{t}) \Xi_{N}^{\theta}+\varepsilon_{N}^{\theta}(\mathbf{t}) .
$$

From this we derive the estimator of $\Xi_{N}^{\theta}$ and consequently the estimate of $\varepsilon_{N}^{\theta}(\mathbf{t})$. We also have the covariance matrix of $\varepsilon_{N}^{\theta}(\mathbf{t})$, defined by

$$
\Sigma_{N}^{\theta}=\left(\sum_{j \geq N+1} \lambda_{j}^{\theta} \varphi_{j}^{\theta}\left(t_{k}\right) \varphi_{j}^{\theta}\left(t_{l}\right)\right)_{\substack{1 \leq k \leq n \\ 1 \leq l \leq n}} .
$$

Since we work with Gaussian process $\varepsilon_{N}^{\theta}$ is gaussian vector, $\varepsilon_{N}^{\theta}(\mathbf{t}) \sim N\left(0, \Sigma_{N}^{\theta}\right)$, and it follows that

$$
\eta_{N}=\left(\Sigma_{N}^{\theta}\right)^{-\frac{1}{2}} \varepsilon_{N}^{\theta}(\mathbf{t}) \sim N\left(0, I_{n}\right)
$$

and then $\left\|\eta_{N}\right\|^{2} \sim \chi^{2}(n)$.

The estimator of $\Xi_{N}^{\theta}$ is

$$
\widehat{\Xi_{N}^{\theta}}=\left(M_{N}^{\theta}(\mathbf{t})^{\prime}\left(\Sigma_{N}^{\theta}\right)^{-1} M_{N}^{\theta}(\mathbf{t})\right)^{-1} M_{N}^{\theta}(\mathbf{t})^{\prime}\left(\Sigma_{N}^{\theta}\right)^{-1} X^{\theta}(\mathbf{t}),
$$


and the estimate of the residuals is $\widehat{\varepsilon_{N}^{\theta}}(\mathbf{t})$ which writes

$$
\widehat{\varepsilon_{N}^{\theta}}(\mathbf{t})=\left(I_{n}-H^{\theta}\right) X^{\theta}(\mathbf{t}),
$$

where $H^{\theta}$ is the hat matrix of the model with the following expression

$$
H^{\theta}=M_{N}^{\theta}(\mathbf{t})\left(M_{N}^{\theta}(\mathbf{t})^{\prime}\left(\Sigma_{N}^{\theta}\right)^{-1} M_{N}^{\theta}(\mathbf{t})\right)^{-1} M_{N}^{\theta}(\mathbf{t})^{\prime}\left(\Sigma_{N}^{\theta}\right)^{-1}
$$

therefore

$$
\widehat{\varepsilon_{N}^{\theta}}(\mathbf{t}) \sim N\left(0,\left(I_{n}-H^{\theta}\right) \Sigma_{N}^{\theta}\left(I_{n}-H^{\theta}\right)^{\prime}\right) .
$$

Now we observe $X^{\theta_{0}}(\mathbf{t})$ with true unknown parameter $\theta_{0}$ and the regression model (5) is given by the expansion on the Karhunen-Loève basis associated to $\theta$. Hence we must write:

$$
X^{\theta_{0}}(t)=\sum_{i \geq 1} \rho_{i}^{\theta, \theta_{0}} \varphi_{i}^{\theta}(t)
$$

where

$$
\rho_{i}^{\theta, \theta_{0}}=\int_{0}^{1} X^{\theta_{0}}(t) \varphi_{i}^{\theta}(t) d t
$$

and when $\theta=\theta_{0}, \rho_{i}^{\theta_{0}, \theta_{0}}=\sqrt{\lambda_{i}^{\theta_{0}}} \xi_{i}^{\theta_{0}}$.

The regression model (5) becomes:

$$
X^{\theta_{0}}(\mathbf{t})=M_{N}^{\theta, \theta_{0}}(\mathbf{t}) \Xi_{N}^{\theta, \theta_{0}}+\varepsilon_{N}^{\theta, \theta_{0}}(\mathbf{t}),
$$

where $M_{N}^{\theta, \theta_{0}}(\mathbf{t})$ and $\Xi_{N}^{\theta, \theta_{0}}$ are:

$$
M_{N}^{\theta, \theta_{0}}(\mathbf{t})=\left(\left\langle X^{\theta_{0}}, \varphi_{j}^{\theta}\right\rangle\right)_{\substack{1 \leq i \leq n \\
1 \leq j \leq N}}, \quad \Xi_{N}^{\theta, \theta_{0}}=\left(\begin{array}{c}
\xi_{1}^{\theta} \\
\vdots \\
\xi_{N}^{\theta}
\end{array}\right)
$$

and

$$
\varepsilon_{N}^{\theta, \theta_{0}}(\mathbf{t})=\left(\varepsilon\left(t_{1}\right)^{\theta, \theta_{0}}, \cdots, \varepsilon\left(t_{n}\right)^{\theta, \theta_{0}}\right)^{\prime}
$$

with

$$
\varepsilon^{\theta, \theta_{0}}\left(t_{i}\right)=\sum_{j \geq N+1}\left\langle X^{\theta_{0}}, \varphi_{j}^{\theta}\right\rangle \varphi_{j}^{\theta}\left(t_{i}\right)=\sum_{j \geq N+1} \rho_{i}^{\theta, \theta_{0}} \varphi_{j}^{\theta}\left(t_{i}\right) .
$$

Our procedure of estimation decomposed in three steps :

1. Observe the real process $X^{\theta_{0}}(\mathbf{t})$ with unknown parameter $\theta_{0}$.

2. Adjust the regression model (8) for $X^{\theta_{0}}(\mathbf{t})$, for "all" values $\theta$ in $(0,1)$.

3. Construct a function based on the estimation of the parameters of the model with the property that discrepancy appears when $\theta \neq \theta_{0}$. 
As previously, the covariance function of the process $\varepsilon_{(t)}^{\theta, \theta_{0}}$ is calculated as:

$$
\begin{aligned}
C^{\theta, \theta_{0}}(t, s) & =\mathbb{E}\left(\varepsilon^{\theta, \theta_{0}}(t) \varepsilon^{\theta, \theta_{0}}(s)\right)=\operatorname{cov}\left(\varepsilon^{\theta, \theta_{0}}(t), \varepsilon^{\theta, \theta_{0}}(s)\right) \\
& =\mathbb{E} \sum_{\substack{i \geq N+1 \\
j \geq N+1}}\left\langle X^{\theta_{0}}, \varphi_{i}^{\theta}\right\rangle\left\langle X^{\theta_{0}}, \varphi_{j}^{\theta}\right\rangle \varphi_{i}^{\theta}(t) \varphi_{j}^{\theta}(s) \\
& =\mathbb{E} \sum_{\substack{i \geq N+1 \\
j \geq N+1}}\left(\int_{0}^{1} \int_{0}^{1} X^{\theta_{0}}(u) X^{\theta_{0}}(v) \varphi_{i}^{\theta}(u) \varphi_{j}^{\theta}(v) d u d v\right) \varphi_{i}^{\theta}(t) \varphi_{j}^{\theta}(s) \\
& =\sum_{\substack{i \geq N+1 \\
j \geq N+1}}\left(\int_{0}^{1} \int_{0}^{1} \Gamma^{\theta_{0}}(u, v) \varphi_{i}^{\theta}(u) \varphi_{j}^{\theta}(v) d u d v\right) \varphi_{i}^{\theta}(t) \varphi_{j}^{\theta}(s),
\end{aligned}
$$

where we recall that $\Gamma^{\theta_{0}}(u, v)=\mathbb{E}\left(X^{\theta_{0}}(u) X^{\theta_{0}}(v)\right)$ is the covariance function for the process $X^{\theta_{0}}(t)$.

If we denote

$$
A_{i, j}^{\theta, \theta_{0}}=\int_{0}^{1} \int_{0}^{1} \Gamma^{\theta_{0}}(u, v) \varphi_{i}^{\theta}(u) \varphi_{j}^{\theta}(v) d u d v,
$$

the final expression for the covariance function of the process $\varepsilon^{\theta, \theta_{0}}(t)$ is

$$
C^{\theta, \theta_{0}}(t, s)=\sum_{\substack{i \geq N+1 \\ j \geq N+1}} A_{i, j}^{\theta, \theta_{0}} \varphi_{i}^{\theta}(t) \varphi_{j}^{\theta}(s) .
$$

Therefore the covariance matrix of $\varepsilon_{N}^{\theta, \theta_{0}}(\mathbf{t})$ is

$$
\Sigma_{N}^{\theta, \theta_{0}}=\left(\sum_{\substack{i \geq N+1 \\ j \geq N+1}} A_{i, j}^{\theta, \theta_{0}} \varphi_{i}^{\theta}\left(t_{k}\right) \varphi_{j}^{\theta}\left(t_{l}\right)\right)_{\substack{1 \leq k \leq n \\ 1 \leq l \leq n}},
$$

Our idea is to use $\Sigma_{N}^{\theta}$ and $\Sigma_{N}^{\theta, \theta_{0}}$ to construct a "contrast function" $h$ that measures the strength of the estimation for all value of $\theta$. This "contrast function" will be built on the residuals of the regression. Actually $h$ will not be a contrast but its derivative, which is enough for our purpose.

So we leave the issue of estimation of the parameters in our model. Let us focus our attention on the random vector of the "standardized" residuals:

$$
\eta_{N}^{\theta, \theta_{0}}=\left(\Sigma_{N}^{\theta}\right)^{-\frac{1}{2}} \varepsilon_{N}^{\theta, \theta_{0}}(\mathbf{t}) \sim N\left(0,\left(\Sigma_{N}^{\theta}\right)^{-\frac{1}{2}} \Sigma_{N}^{\theta, \theta_{0}}\left(\Sigma_{N}^{\theta}\right)^{-\frac{1}{2}}\right) .
$$

This involves the actual covariance matrix (unknown) and the covariance matrix for a model depending on $\theta$, and we have:

$$
\mathbb{E}\left\|\eta_{N}^{\theta, \theta_{0}}\right\|^{2}=\mathbb{E}\left(\varepsilon_{N}^{\theta, \theta_{0}}(\mathbf{t})^{\prime}\left(\Sigma_{N}^{\theta}\right)^{-1} \varepsilon_{N}^{\theta, \theta_{0}}(\mathbf{t})\right)=\operatorname{tr}\left(\Sigma_{N}^{\theta, \theta_{0}}\left(\Sigma_{N}^{\theta}\right)^{-1}\right)
$$


Then we define the contrast function $h_{N}$ by:

$$
h_{N}\left(\theta ; \theta_{0}\right)=\lim _{n \rightarrow+\infty} \frac{\operatorname{tr}\left(\Sigma_{N}^{\theta, \theta_{0}}\left(\Sigma_{N}^{\theta}\right)^{-1}\right)}{n}=\lim _{n \rightarrow+\infty} \frac{\mathbb{E}\left\|\eta_{N}^{\theta, \theta_{0}}\right\|^{2}}{n}
$$

If $\theta=\theta_{0}$, then $\Sigma_{N}^{\theta_{0}, \theta_{0}}=\Sigma_{N}^{\theta_{0}}, \eta_{N}^{\theta_{0}, \theta_{0}}=\eta_{N}$ and $\frac{\operatorname{tr}\left(\Sigma_{N}^{\theta_{0}, \theta_{0}}\left(\Sigma_{N}^{\theta_{0}}\right)^{-1}\right)}{n}=1$, so that $h_{N}\left(\theta_{0} ; \theta_{0}\right)=1$.

Furthermore we know the behaviour of $h_{N}$ when $\theta \neq \theta_{0}$ :

\section{Theorem 2.1.}

We assume that $X^{\theta}(t)$ is a fractional Brownian motion or a fractional Ornstein-Ulhbeck with Hurst parameter $\theta \in(0,1)$ defined on $[0,1]$ and $X^{\theta_{0}}(\mathbf{t})$ for $\mathbf{t}=\left(t_{1}, \cdots, t_{n}\right)$ is the partial observation of a path of the process $X^{\theta_{0}}$. The times of observation $\mathbf{t}$ are assumed to be equally spaced or random i.i.d. variables uniformly distributed on $[0,1]$, independent of $X^{\theta_{0}}$. The function

$$
h_{N}\left(\theta ; \theta_{0}\right)=\lim _{n \rightarrow+\infty} \frac{\operatorname{tr}\left(\Sigma_{N}^{\theta, \theta_{0}}\left(\Sigma_{N}^{\theta}\right)^{-1}\right)}{n}
$$

with $\Sigma_{N}^{\theta}$ and $\Sigma_{N}^{\theta, \theta_{0}}$ defined in (6) and (11) respectively satisfies the following property:

$$
\begin{aligned}
& \checkmark \quad \text { If } \theta=\theta_{0} \text { then } h_{N}\left(\theta ; \theta_{0}\right)=1 . \\
& \checkmark \quad \text { If } \theta>\theta_{0} \text { then } h_{N}\left(\theta ; \theta_{0}\right)=\infty . \\
& \checkmark \quad \text { If } \theta<\theta_{0} \text { then } h_{N}\left(\theta ; \theta_{0}\right)=0 .
\end{aligned}
$$

The proof is postponed in Annex A.

The previous result is obtained in a rather theoretical setting : the Karhunen-Loève decomposition is known, $N$ is fixed and the limit $n \rightarrow \infty$ is taken. In practice we have to choose $N$, use an approximation of the Karhunen-Loève basis, $n$ is finite, we only can compute estimations of $h_{N}$, and finally it is not possible to estimate $h_{N}$ at every possible value of $\theta_{0}$. The question of the choice of $N$ will be studied by simulation.

Now we estimate $h_{N}$. We use:

$$
\widehat{h}_{N}\left(\theta ; \theta_{0}\right)=\frac{\left\|\widehat{\eta_{N}^{\theta, \theta_{0}}}\right\|^{2}}{n-N}=\frac{\left(\widehat{\varepsilon_{N}^{\theta, \theta_{0}}}(\mathbf{t})^{\prime}\left(\Sigma_{N}^{\theta}\right)^{-1} \widehat{\varepsilon_{N}^{\theta, \theta_{0}}}(\mathbf{t})\right)}{n-N}
$$

where

$$
\widehat{\varepsilon_{N}^{\theta, \theta_{0}}}(\mathbf{t})=\left(I_{n}-H^{\theta}\right) \varepsilon_{N}^{\theta, \theta_{0}}(\mathbf{t}) \sim N\left(0,\left(I_{n}-H^{\theta}\right) \Sigma_{N}^{\theta, \theta_{0}}\left(I_{n}-H^{\theta}\right)^{\prime}\right) .
$$

Conditionally to the time values $\mathbf{t},\left\|\widehat{\eta_{N}^{\theta_{0}, \theta_{0}}}\right\|^{2}$ is a $\chi^{2}$ with $n-N$ degrees of freedom, so that $\mathbb{E} \widehat{h}_{N}\left(\theta_{0} ; \theta_{0}\right)=1$ and $\widehat{h}_{N}\left(\theta_{0} ; \theta_{0}\right)$ converges in probability toward 1 .

Hence we propose to estimate the parameter $\theta_{0}$ by :

$$
\widehat{\theta}=\operatorname{Argmin}_{\theta}\left|\widehat{h}_{N}\left(\theta ; \theta_{0}\right)-1\right|
$$


To compute this estimator we need an approximation of the Karhunen-Loève basis. For this we built a database with the values of the covariance matrix $\Gamma^{\theta}(\mathbf{t}, \mathbf{s})$ for a suitable collection of $\theta$ on a discretization of $[0,1]$ and for each fractional process we studied. It remains to numerically computatie $\Sigma^{1}$ which is possible even for about thousand points. We avoid the term $\log (\operatorname{det} \Sigma)$ that appears in the M.L.E. estimation.

As we focus onto the method itself we do not discuss the numerical implementation of this data basis, but we easily obtain good numerical approximations by simulation, and we do it only once for all.

Then $\widehat{\theta}$ is computed by interpolation of the grid values.

\subsubsection{Simulation Study and Numerical Results}

\subsubsection{Fractional Brownian}

We conduct a simulation study to compare the estimates from our method (KL method) with the M.L.E.. Both procedures were used to give estimates of the Hurst parameter in fractional processes. This study shows that the KL method performs well.

The simulation study has been organized in two parts

1. A first simulation study to find a good choice of $N$ when $n$ is fixed and the same for $n$ for $N$ fixed (it is reasonable order).

2. A simulation study with good choices of $n$ and $N$ in order to find the estimation of the Hurst parameter for the fractional processes.

For the Fractional Brownian motion we set

- $n=300$,

- $N=5 ; 10 ; 30 ; 50 ; 100 ; 150 ; 200$, and

- $\theta_{0}=0.1$ and $\theta_{0}=0.7$.

The first values of $\theta_{0}$ is close to 0 and is not easy to estimate. The second one illustrate an easier case. We tried many values of $N$, but we knew that only small values (5 to 10 ) would be of practical use.

The value $n=300$ is in the range of easy computation of the M.L.E..

For each combination of previous values we generate 50 random samples of the fractional process. Then we calculate the value of $\widehat{h}\left(\theta ; \theta_{0}\right)$ on a grid of values of $\theta$ from 0.05 to 0.95 by step of 0.01 . Finally we interpolate the results obtained to compute $\widehat{\theta}_{0}$ for which $\widehat{h}\left(\theta ; \theta_{0}\right)=1$. We use a parabolic interpolation because of the behavior of $\widehat{h}$.

Table 1 shows that the KL method gives good results for any value of $N$. This is confirmed observing the box-plot representation for each case (see Figure ??). However, it is important to highlight that for the greatest values of $N$ more variability in the estimations is observed.Thus, using small values of $N$ produce best results for estimating $\theta_{0}$. Here it is worth pointing out that the theoretical assumption needed for $N(N \gg 1)$ can be relaxed in numerical calculations. This make our KL method easily tractable in any case. In the second part of the simulation study we analyze of the behavior of $\widehat{\theta}$ with $n$ when $N$ is fixed.

In the light of the previous simulation study we consider the following settings. 


\begin{tabular}{|c|c|c|}
\hline $\mathbf{N}$ & Mean & STD \\
\hline 5 & 0,1018 & 0,0078 \\
\hline 10 & 0,1018 & 0,0075 \\
\hline 30 & 0,1018 & 0,0074 \\
\hline 50 & 0,1021 & 0,0071 \\
\hline 100 & 0,1023 & 0,0083 \\
\hline 150 & 0,1027 & 0,0094 \\
\hline 200 & 0,1038 & 0,0115 \\
\hline
\end{tabular}

\begin{tabular}{|c|c|c|}
\hline $\mathbf{N}$ & Mean & STD \\
\hline 5 & 0,7004 & 0,0060 \\
\hline 10 & 0,7004 & 0,0060 \\
\hline 30 & 0,7003 & 0,0061 \\
\hline 50 & 0,7001 & 0,0061 \\
\hline 100 & 0,7002 & 0,0075 \\
\hline 150 & 0,7010 & 0,0083 \\
\hline 200 & 0,7025 & 0,0091 \\
\hline
\end{tabular}

Table 1: Mean and standard deviation for the estimation of $\theta_{0}$ for each value of $N$ by using 50 random observations of the FBm with the true parameter and $n=300$. (Left: $\theta_{0}=0.1$. Right: $\theta_{0}=0.7$ ).

- $N=5$,

- $n=10 ; 50 ; 100 ; 150 ; 200 ; 300$, and

- $\theta_{0}=0.1$ and $\theta_{0}=0.7$.

For each combination of the previous values we generate 50 random samples of the fractional process. Then we calculate the value of $\widehat{h}\left(\theta ; \theta_{0}\right)$ as described previously.

\begin{tabular}{|c|c|c|}
\hline $\mathbf{n}$ & Mean & STD \\
\hline 10 & 0,1236 & 0,0377 \\
\hline 50 & 0,0828 & 0,0451 \\
\hline 100 & 0,1025 & 0,0167 \\
\hline 150 & 0,1006 & 0,0119 \\
\hline 200 & 0,0977 & 0,0094 \\
\hline 300 & 0,0997 & 0,0071 \\
\hline
\end{tabular}

\begin{tabular}{|c|c|c|}
\hline $\mathbf{n}$ & Mean & STD \\
\hline 10 & 0,7200 & 0,0349 \\
\hline 50 & 0,6848 & 0,1007 \\
\hline 100 & 0,7029 & 0,0122 \\
\hline 150 & 0,7005 & 0,0082 \\
\hline 200 & 0,6992 & 0,0075 \\
\hline 300 & 0,6991 & 0,0064 \\
\hline
\end{tabular}

Table 2: Mean and standard deviation for the estimation of $\theta_{0}$ for each value of $n$ by using 50 random observations of the FBm with the true parameter and $N=5$. (Left: $\theta_{0}=0.1$. Right: $\theta_{0}=0.7$ ).

The numerical results in Tables 2 and Figure ?? allows to thinkthat our estimator converges, and that the speed of convergence is of the correct magnitude.

Therefore we conclude that a choice of $N$ between 5 and 10 is appropriate. if we a priori suspect small value of $\theta_{0} N=10$ would be enough since the eigenvalues of the covariance operator decrease slowly, a contrario when $\theta_{0}$ is large the eigen values decreases faster and $N=5$ would be better

According to the previous conclusions we perform a last simulation study to compare the performances of the KL method and the M.L.E..

The study provides quantitative results for different values of the parameter. For the simulation scheme we set $\theta_{0} \in\{0.1,0.3,0.7,0.9\}, n=200, N=10$ for the first two values of $\theta_{0}$ and $N=5$ for the other two.

For the M.L.E. we maximize the:

$$
\widehat{h}_{m l e}\left(\theta ; \theta_{0}\right)=-\ln \left(\operatorname{det}\left(D_{n}^{\theta_{0}} \Gamma^{\theta}(\mathbf{t})\right)\right)-\left(X_{\mathbf{t}}^{\theta_{0}}\right)^{\prime} \Gamma^{\theta}(\mathbf{t}) X_{\mathbf{t}}^{\theta_{0}},
$$


where $\Gamma^{\theta}(\mathbf{t})=\left(\Gamma^{\theta}\left(t_{i}, t_{j}\right)\right)_{1 \leq i, j \leq n}, D_{n}^{\theta_{0}}$ is the function who assure that $\operatorname{det}\left(D_{n}^{\theta_{0}} \Gamma^{\theta}(\mathbf{t})\right)>0$ in the numerical implementation. The matrix $\Gamma^{\theta}(\mathbf{t})$ is definite positive for any $\mathbf{t}$ hence $\operatorname{det}\left(\Gamma^{\theta}(\mathbf{t})\right)>0$. But due to the small values of the eigenvalues associated to $\Gamma^{\theta}(\mathbf{t})$ we faced numerical difficulties. We easily avoided this numerical problem.

For both methods we improved the numerical precision by a quadratic interpolation in each case. The results can be seen in Table 3.

\begin{tabular}{|c|c|c|c|c|}
\hline & Mean & STD & Mean & STD \\
\hline 0.1 & 0,1004 & 0,0100 & 0,0993 & 0,0119 \\
\hline 0.3 & 0,3009 & 0,0085 & 0,2998 & 0,0078 \\
\hline 0.7 & 0,7008 & 0,0082 & 0,7007 & 0,0081 \\
\hline 0.9 & 0,9004 & 0,0051 & 0,9007 & 0,0081 \\
\hline
\end{tabular}

Table 3: Mean and standard deviation for the estimation of several $\theta_{0}$.

The results of table 3 clearly prove that the two methods are are numerically equivalent. Of curse the M.L.E. estimator is the by the Cramer Rao bound, but the KL method provides an almost as precise estimator as the M.L.E. This partial conclusion for the Fractional Brownian motion has been confirmed by the study of fractionnal ornstein-ulhlhenbeck(F.O.U.) of the third kind. 


\subsubsection{Fractional Ornstein-Uhlenbeck. First Kind}

For this case has not interest in perform a simulation study. As we state before we need to construct a FBm using the FOU of the first kind and conduct the KL method to the obtained FBm. In that sense we use the inverse of

$$
X_{t_{n}}^{\theta_{0}, 1} \approx e^{-\widetilde{\theta} t_{n}}\left(X_{t_{0}}^{\theta_{0}, 1}+\sigma \sum_{j=1}^{n} e^{-\widetilde{\theta} t_{j}} \Delta W_{t_{n}}^{\theta_{0}}\right)
$$

hence if $X_{t_{i}}^{\theta_{0}, 1}$ is observed we get

$$
W_{t_{n}}^{\theta_{0}} \approx W_{t_{n-1}}^{\theta_{0}}+X_{t_{n}}^{\theta_{0}, 1}-e^{-\widetilde{\theta} \Delta_{n}} X_{t_{n-1}}^{\theta_{0}, 1}
$$

Obviously for the simulation study we construct first the FBm to obtain the FOU of first kind. Therefore the reversal formula is used. It is an obvious consequence that we will obtain the same FBm that was generated. Hence is not necessary to conduct a simulation study because in this case the general setting for the FBm applies.

\section{Annex A : proof of theorem 2.1}

We suppose $\theta \neq \theta_{0}$.

We begin by taking "approximations" of the matrices $\Sigma_{N}^{\theta}, \Sigma_{N}^{\theta, \theta_{0}}$. Let $N^{\star}$ we define the following matrices in order to approximate $\Sigma_{N}^{\theta, \theta_{0}}, \Sigma_{N}^{\theta}$ :

$$
\Psi_{N^{\star}}=\left(\varphi_{j}^{\theta}\left(t_{i}\right)\right)_{\substack{1 \leq i \leq n \\ N+1 \leq j \leq N^{\star}}} A_{N^{\star}}=\left(A_{i, j}^{\theta, \theta_{0}}\right)_{\substack{N+1 \leq i \leq N^{\star} \\ N+1 \leq j \leq N^{\star}}} \Lambda_{N^{\star}}=\left(\lambda_{i j}^{\theta}\right)_{\substack{N+1 \leq i \leq N^{\star} \\ N+1 \leq j \leq N^{\star}}},
$$

where $\lambda_{i j}^{\theta}=\lambda_{i}^{\theta}$ if $i=j$ and zero otherwise. Therefore we have the following approximations:

$$
\Sigma_{N, N^{\star}}^{\theta}=\Psi_{N^{\star}} \Lambda_{N^{\star}} \Psi_{N^{\star}}^{\prime} \quad \text { and } \quad \Sigma_{N, N^{\star}}^{\theta, \theta_{0}}=\Psi_{N^{\star}} A_{N^{\star}} \Psi_{N^{\star}}^{\prime}
$$

Since $\operatorname{tr}(A B)=\operatorname{tr}(B A)$ for any matrices $A, B$ such as $A B$ and $B A$ are square matrices it follows that

$$
\begin{aligned}
\operatorname{tr}\left(\Sigma_{N^{\prime} N^{\star}}^{\theta, \theta_{0}}\left(\Sigma_{N, N^{\star}}^{\theta}\right)^{-1}\right) & =\operatorname{tr}\left(\Psi_{N^{\star}} A_{N^{\star}} \Psi_{N^{\star}}^{\prime}\left(\Psi_{N^{\star}} \Lambda_{N^{\star}} \Psi_{N^{\star}}^{\prime}\right)^{-1}\right) \\
& =\operatorname{tr}\left(A_{N^{\star}} \Lambda_{N^{\star}}^{-1}\right)
\end{aligned}
$$

Assuming $N^{\star} \geq n+N$ and large enough, $\operatorname{rank}\left(\Psi_{N^{\star}}^{\prime}\right)=n$ (a.s. if $\mathbf{t}$ is random) and the matrix

$$
\Lambda_{N^{\star}}^{1 / 2} \Psi_{N^{\star}}^{\prime}\left(\Psi_{N^{\star}} \Lambda_{N^{\star}} \Psi_{N^{\star}}^{\prime}\right)^{-1} \Psi_{N^{\star}} \Lambda_{N^{\star}}^{1 / 2}
$$

is the matrix of the orthogonal projection on the column of $\Lambda_{N^{\star}}^{1 / 2} \Psi_{N^{\star}}^{\prime}$. Hence the matrix $A_{N^{\star}} \Psi_{N^{\star}}^{\prime}\left(\Psi_{N^{\star}} \Lambda_{N^{\star}} \Psi_{N^{\star}}^{\prime}\right)^{-1} \Psi_{N^{\star}}$ is conjugate with $\Lambda_{N^{\star}}^{-1 / 2} A_{N^{\star}} \Lambda_{N^{\star}}^{-1 / 2}$. We have obtained:

$$
\operatorname{tr}\left(\Sigma_{N, N^{\star}}^{\theta, \theta_{0}}\left(\Sigma_{N, N^{\star}}^{\theta}\right)^{-1}\right)=\operatorname{tr}\left(A_{N^{\star}} \Lambda_{N^{\star}}^{-1}\right)=\sum_{i \geq N+1}^{N^{\star}} \frac{A_{i, i}^{\theta, \theta_{0}}}{\lambda_{i}^{\theta}} .
$$




\subsubsection{Fractional Brownian motion (fBm)}

In this case we will use the results of Bronski (?). This article provides expansion of the eigenvalues appearing in the Karhunen-Loève decomposition of the $\mathrm{fBm}$, more precisely

$$
\lambda_{n}^{\theta}=\frac{v_{\theta}}{n^{2 \theta+1}}+o\left(n^{-\frac{(2 \theta+2)(4 \theta+3)}{4 \theta+5}+\delta}\right) \quad \forall \delta>0, \quad n \gg 1,
$$

where

$$
v_{\theta}=\frac{\sin (\pi \theta) \Gamma(2 \theta+1)}{\pi^{2 \theta+1}},
$$

and $\Gamma$ is the usual Gamma function.

In the proof of the previous result the author define an integral operator $\mathbf{A}$ in the orthonormal basis $\left\{\sqrt{2} \sin \left(n+\frac{1}{2}\right) \pi x\right\}_{n=0}^{\infty}$, which is the Karhunen-Loève basis of the Wiener process. The $\mathbf{A}_{m, n}$ element for $\theta=\theta_{0}$ writes:

$$
\mathbf{A}_{m, n}=\int_{0}^{1} \int_{0}^{1}\left(u^{2 \theta_{0}}+v^{2 \theta_{0}}-|u-v|^{2 \theta_{0}}\right) \sin \left(m^{*} u\right) \sin \left(n^{*} v\right) d u d v
$$

where $n^{*}=(n+1 / 2) \pi$ and likewise $m^{*}$.

Bronski shows that $\mathbf{A}$ can be written as $\mathbf{A}=\mathbf{D}+\mathbf{O}$, where $\mathbf{D}$ is a diagonal piece and $\mathbf{O}$ an off-diagonal piece (the expressions for both are in ?). In our case is easy to see that

$$
A_{i, j}^{1 / 2, \theta_{0}}=\mathbf{A}_{i, j} .
$$

We have to study the quotient $A_{i, i}^{\theta, \theta_{0}} / \lambda_{i}^{\theta}$, hence we focus on the diagonal entries of $\mathbf{A}$. Therefore it follows

$$
A_{n, n}^{1 / 2, \theta_{0}}=\frac{\sin \left(\pi \theta_{0}\right) \Gamma\left(2 \theta_{0}+1\right)}{n^{* 2 \theta_{0}+1}}+O\left(n^{-2\left(\theta_{0}+1\right)}\right),
$$

thus

$$
\frac{A_{n, n}^{1 / 2, \theta_{0}}}{\lambda_{n}^{1 / 2}}=\sin \left(\pi \theta_{0}\right) \Gamma\left(2 \theta_{0}+1\right) n^{2 \theta_{0}-1}+O\left(\frac{(n+1 / 2)^{2}}{n^{2\left(\theta_{0}+1\right)}}\right) .
$$

Now it is straightforward that

$$
\lim _{n \rightarrow+\infty} \frac{1}{n} \sum_{i \geq N+1}^{n+N} \frac{A_{i, i}^{1 / 2, \theta_{0}}}{\lambda_{i}^{1 / 2}}=\left\{\begin{aligned}
+\infty & \text { if } \theta_{0}>1 / 2 \\
0 & \text { if } \theta_{0}<1 / 2
\end{aligned}\right.
$$

With this result we just get the result in the case $\theta=1 / 2$, but the general follow the same way. In Bronski (?) is established an asymptotic expansion of the eigenvalues and eigenfunctions of the FBm

$$
\begin{aligned}
\lambda_{n}^{\theta} & \sim \frac{v_{\theta}}{n^{2 \theta+1}} \quad n \gg 1, \\
\varphi_{n}^{\theta}(x) & \sim \sqrt{2} \sin \left(n^{*} x\right) \quad n \gg 1
\end{aligned}
$$


Hence setting

$$
\widetilde{\mathbf{A}}_{m, n}=\int_{0}^{1} \int_{0}^{1}\left(u^{2 \theta_{0}}+v^{2 \theta_{0}}-|u-v|^{2 \theta_{0}}\right) \varphi_{n}^{\theta}(u) \varphi_{n}^{\theta}(v) d u d v
$$

and using (14) we obtain

$$
A_{n, n}^{\theta, \theta_{0}}=\widetilde{\mathbf{A}}_{n, n} \sim \mathbf{A}_{n, n}=\frac{\sin \left(\pi \theta_{0}\right) \Gamma\left(2 \theta_{0}+1\right)}{n^{* 2 \theta_{0}+1}}+O\left(n^{-2\left(\theta_{0}+1\right)}\right) .
$$

That leads us to

$$
\frac{A_{n, n}^{\theta, \theta_{0}}}{\lambda_{n}^{\theta}} \sim \sin \left(\pi \theta_{0}\right) \Gamma\left(2 \theta_{0}+1\right) v_{\theta}^{-1} \frac{n^{2 \theta+1}}{n^{* 2 \theta_{0}+1}}+O\left(\frac{n^{2 \theta+1}}{n^{2\left(\theta_{0}+1\right)}}\right) .
$$

Therefore

$$
\lim _{n \rightarrow+\infty} \frac{1}{n} \sum_{i \geq N+1}^{n+N} \frac{A_{i, i}^{\theta, \theta_{0}}}{\lambda_{i}^{\theta}}=\left\{\begin{array}{rl}
+\infty & \text { if } \theta_{0}>\theta \\
0 & \text { if } \theta_{0}<\theta
\end{array} .\right.
$$

This ends the proof for the fBm.

\subsubsection{Fractional Ornstein-Uhlenbeck (First Kind)}

Let $X_{t}^{\theta, 1}$ be the Fractional Ornstein-Uhlenbeck (fOU) of first kind (see (??)). It is the solution of the Stochastic Differential Equation driven by a fractional Brownian motion

$$
d X_{t}^{H, 1}=-\alpha X_{t}^{\theta, 1} d t+\sigma d W_{s}^{\theta}
$$

Here we assume that $\alpha$ and $\sigma$ are known.

There is no closed form for the covariance function or its eigenvalues, but one can relate them to the corresponding values for the $\mathrm{fBm}$.

Let us denote $\Gamma^{\theta, B},\left(\varphi_{j}^{\theta, B}(t)\right)_{j \geq 1}$ and $\left(\lambda_{j}^{\theta, B}\right)_{j \geq 1}$ the covariance function, KL eigenfunctions and eigenvalues respectively for the fBm. The process $X_{t}^{\theta, 1}$ can be expanded on $\left(\varphi_{j}^{\theta, B}(t)\right)_{j \geq 1}$, which is an orthonormal basis of $\mathcal{L}^{2}([0,1])$. Then

$$
X^{\theta_{0}, 1}(t)=\sum_{i \geq 1} \rho_{i}^{\theta, \theta_{0}} \varphi_{i}^{\theta, B}(t)
$$

where

$$
\rho_{i}^{\theta, \theta_{0}}=\int_{0}^{1} X^{\theta_{0}, 1}(t) \varphi_{i}^{\theta, B}(t) d t
$$

therefore the expression for $A_{i j}^{\theta, \theta_{0}}$ in (9) still takes the form

$$
A_{i, j}^{\theta, \theta_{0}}=\int_{0}^{1} \int_{0}^{1} \Gamma^{\theta_{0}}(u, v) \varphi_{i}^{\theta, B}(u) \varphi_{j}^{\theta, B}(v) d u d v
$$


where $\Gamma^{\theta_{0}}$ is the covariance function of the fOU. We have:

$$
\Sigma_{N}^{\theta, \theta_{0}}=\left(\sum_{\substack{i \geq N+1 \\ j \geq N+1}} A_{i, j}^{\theta, \theta_{0}} \varphi_{i}^{\theta, B}\left(t_{k}\right) \varphi_{j}^{\theta, B}\left(t_{l}\right)\right)_{\substack{1 \leq k \leq n \\ 1 \leq l \leq n}} .
$$

As in (??) we have:

$$
h_{N}\left(\theta ; \theta_{0}\right)=\lim _{n \rightarrow+\infty} \frac{1}{n} \sum_{i \geq N+1}^{n+N} \frac{A_{i, i}^{\theta, \theta_{0}}}{\lambda_{i}^{\theta}} .
$$

The inequality for the covariance function of the fOU in(??) yields:

$$
\begin{gathered}
c_{\theta}^{\prime} A_{i, i}^{\theta, \theta_{0}, B} \leq A_{i, i}^{\theta, \theta_{0}} \leq C_{\theta}^{\prime} A_{i, i}^{\theta, \theta_{0}, B} \\
d_{\theta}^{\prime} \lambda_{i}^{\theta, B} \leq \lambda_{i}^{\theta} \leq D_{\theta}^{\prime} \lambda_{i}^{\theta, B}
\end{gathered}
$$

where the constants $c_{\theta}^{\prime}, C_{\theta}^{\prime}, d_{\theta}^{\prime}, D_{\theta}^{\prime}$ are positive. Hence

$$
c_{\theta}^{\prime \prime} h_{N}^{B}\left(\theta ; \theta_{0}\right) \leq h_{N}\left(\theta ; \theta_{0}\right) \leq C_{\theta}^{\prime \prime} h_{N}^{B}\left(\theta ; \theta_{0}\right)
$$

and finally

$$
\lim _{n \rightarrow+\infty} \frac{1}{n} \sum_{i \geq N+1}^{n+N} \frac{A_{i, i}^{\theta, \theta_{0}}}{\lambda_{i}^{\theta}}=\left\{\begin{array}{rl}
+\infty & \text { if } \theta_{0}>\theta \\
0 & \text { if } \theta_{0}<\theta
\end{array},\right.
$$

Again we note the spatial scale invariance of our estimator. Yet there still remain another unknown parameter $\alpha$.

\subsubsection{Somme comments about the fractional Ornstein-Uhlenbeck (Second and third Kind)}

The process $X^{\theta, 2}$, fOU of the second kind, satisfies:

$$
X_{t}^{\theta, 2}=e^{-\theta t} W_{a_{t, \theta}}^{\theta}
$$

where $a_{t, \theta}=\theta \sigma^{2} e^{\theta t / \theta} / \theta$, thus we propose a different approach. We use the previous equation to revert the fractional Process of second kind into a fractional Brownian motion. This method can also be used for the fOU of first kind. For both cases we can construct a FBm $W^{\theta}(\mathbf{t})$ with Hurst parameter $\theta \in(0,1)$ in $\mathbf{t}=\left(t_{1}, \cdots, t_{n}\right)$ and $\mathbf{t}=\left(e^{\theta t_{1} / H}, \cdots, e^{\theta t_{n} / H}\right)$ respectively. Theorem ?? applies with the correct modifications for the fOU of second kind. This assertion state that a correct estimation of the Hurst parameter on a fBm leads us to a correct estimation for the associated fOU.

For the FOU of third kind $X^{\theta, 3}(t)$ we know the exact expression of the covariance function. Hence the same procedure can be used. However,due to Rosenblatt, we only have asymptotic for the eigenvalues:

$$
\lambda_{n}^{\theta, 3} \sim n^{-(1+2 \theta)},
$$

we refer the reader to ? and ? for details. Therefore we are not in position to give a proof of our theorem for this process. 